Recomendación sobre la medida de la resistividad eléctrica en probetas de hormigón

\title{
Recomendaciones Técnicas
}

\section{Carmen Andrade Perdrix Miguel Ángel Climent Llorca}

https://oi.org/10.21041/AlconpatInternacional/RecTec/2020-04-resistividadenprobetas

\section{ALCONPAT Internacional}

Asociación Latinoamericana de Control de Calidad, Patología y Recuperación de la Construcción

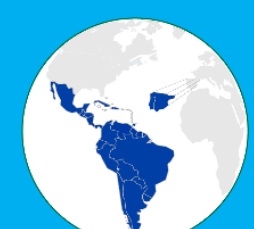




\section{ReCOMENDACIÓN TÉCNICA}

Editores

Pedro Garcés Terradillos

Director de Recomendaciones Técnicas

Universidad de Alicante, España
Jorge Alberto Briceño Mena

Subdirector de Recomendaciones Técnicas

Cinvestav del IPN, Unidad Mérida, México

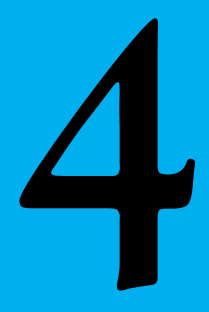

2020

Recomendación sobre la medida de la resistividad eléctrica en probetas de hormigón

DOI: https://doi.org/10.21041/AlconpatInternacional/RecTec/2020-04resistividadenprobetas

Recomendações sobre a medição da resistividade elétrica em corpos de prova de concreto

Recommendations on the measurement of electrical resistivity in concrete specimens

\section{Carmen Andrade Perdrix}

Centro Internacional de Métodos Numéricos en Ingeniería, Madrid, España.

\section{Miguel Ángel Climent Llorca}

Universidad de Alicante, Alicante, España. 


\section{Recomendación Técnica No. 4}

\section{MEDIDA DE LA RESISTIVIDAD ELÉCTRICA EN PROBETAS}

\section{OBJETIVO}

Esta Recomendación Técnica tiene como objeto describir dos métodos de medida de la resistividad eléctrica del hormigón en probetas, tanto cúbicas como cilíndricas y en testigos extraídos de obra construida: el método directo y el método de los cuatro electrodos, o de Wenner.

\section{RESUMEN Y PRINCIPIOS DEL MÉTODO}

Como indica la Ley de Ohm la resistividad es una propiedad volumétrica del material que representa la resistencia eléctrica específica del material, es decir la resistencia al paso de la corriente eléctrica por unidad de volumen (se considera una muestra del material de $1 \mathrm{~m}^{2}$ de sección transversal y $1 \mathrm{~m}$ de espesor). $\mathrm{La}$ resistividad es la inversa de la conductividad. La resistividad eléctrica del hormigón es un indicador de su durabilidad. Es también una medida indirecta de su porosidad y de su grado de saturación en agua. Así, cuanto mayor es la resistividad menor es la porosidad del hormigón y mayor su resistencia mecánica, al tener más fase sólida por volumen. Un hormigón presenta el mínimo de su resistividad cuando está saturado de agua. Cuando el hormigón se seca, su resistividad aumenta exponencialmente.

Dado que la mayoría de los áridos no conducen la corriente eléctrica. La resistividad es la resultante de la conducción electrolítica a través de los poros de la pasta de cemento. Es necesario prestar atención a pesar de ello a la posibilidad de contribución de los áridos, si estos son porosos, o tienen sustancias conductoras de la corriente.
También es necesario tener en cuenta la presencia de fibras conductoras en el hormigón.

La resistividad del hormigón también depende de su temperatura, por lo que suele usarse una temperatura como referencia. A efectos de esta Recomendación Técnica la temperatura de referencia es la de $20 \pm 2^{\circ} \mathrm{C}$.

Dado que al mezclar el cemento con el agua se obtiene una suspensión, la Resistividad al principio del mezclado es muy baja, pero, según se va hidratando el cemento y el hormigón va fraguando y endureciendo, la resistividad aumenta y este aumento es paralelo al de la resistencia mecánica. La velocidad de aumento de la resistividad con el tiempo se ha definido como el "factor de edad" que es el exponente "q" de la ecuación [1], que se debe ajustar a la curva de evolución en el tiempo de cada hormigón particular:

$$
\rho_{t}=\rho_{0}\left(t / t_{0}\right)^{q}
$$

donde $\rho_{t}=$ resistividad en el tiempo t, $\rho_{0}$ es la resistividad en la primera medida (normalmente a los 28 días ( $\left.\mathrm{t}_{0}\right)$ en curado saturado). 


\section{NORMAS DE CONSULTA}

Existe una Norma UNE que describe los dos métodos de medida que contempla esta Recomendación Técnica: PNE 83988 - Durabilidad del hormigón - Determinación de la resistividad del hormigón Parte 1 (Método directo) y Parte 2 (Método de Wenner).

\section{DEFINICIONES Y APARATOS NECESARIOS}

\subsection{Definiciones}

\section{Resistencia eléctrica $\left(\mathbf{R}_{\mathrm{e}}\right)$}

Es la relación entre la diferencia de potencial (V) entre dos electrodos y la intensidad de corriente (I) que circula por el interior de la probeta o testigo, ecuación [2].

$$
R_{e}=\frac{V}{I}
$$

\section{Resistividad eléctrica ( $\rho)$}

Es la Resistencia eléctrica de la unidad de volumen del material. Se obtiene de la relación entre la diferencia de potencial y la intensidad de corriente circulante referida a una geometría normalizada (cubo de $1 \mathrm{~m}$ de arista).

$$
R=\frac{V}{I}=\rho \cdot \frac{L}{A}
$$

- $\quad$ R es la resistencia eléctrica del hormigón en $\Omega$.

- I es la Intensidad eléctrica que circula por el circuito en A.

- V es la diferencia de potencial entre los electrodos en $\mathrm{V}$.

- $\quad \rho$ es resistividad en ohm-cm u ohm-m.

- L es la distancia entre los electrodos de potencial

- A el área transversal de paso de la corriente.
Resistividad volumétrica $\left(\rho_{\mathrm{v}}\right)$

Se formula como:

$$
\rho_{v}=R e \cdot F_{g v}=R e \cdot \frac{A}{L}
$$

\section{Resistividad superficial-medio} infinito $\left(\rho_{s, \text { inf }}\right)$

Se formula como:

$$
\rho_{\operatorname{sinf}}=R e \cdot F_{g s}=R_{\theta} \cdot 2 \cdot \pi \cdot a
$$

La resistividad superficial-medio infinito es igual a la resistividad volumétrica si la medida se realiza sobre una superficie de dimensiones suficientemente grandes como para que se cumpla la condición física de medio quasiinfinito.

\section{Resistividad superficial-medio finito $\left(\rho_{s}\right)$}

Se formula como:

$$
\rho_{s}=\rho_{s, i n f} \cdot F_{f}=\rho_{v} \quad[6]
$$

\section{Factores geométricos $\left(\mathrm{F}_{\mathrm{g}}\right)$}

El factor geométrico es la relación entre la resistividad y la resistencia eléctrica.

En el método volumétrico se denomina $\left(\mathrm{F}_{\mathrm{gv}}\right)$ y es el Área (A) dividida por la distancia entre los electrodos (L) que miden el potencial. 


$$
F_{g v}=\frac{A}{L}
$$

En el método superficial el factor geométrico $\left(\mathrm{F}_{\mathrm{gs}}\right)$ para medio infinito:

$$
F_{g s}=2 \cdot \pi \cdot a \quad[8]
$$

donde "a" es la distancia entre los electrodos, siempre que estén todos igualmente espaciados. Este factor hace que la resistividad superficial medio-infinito $\left(\rho_{\mathrm{s}, \text { inf }}\right)$ resulte el mismo valor que la medida volumétrica $\left(\rho_{\mathrm{v}}\right)$.

\section{Factor de forma $\left(F_{f}\right)$}

Se denomina factor de forma $\left(\mathrm{F}_{\mathrm{f}}\right)$ al que en la medida superficial tiene en cuenta que el medio sea finito y no infinito. Es pues el factor que convierte el valor de la medida superficial de la resistividad en medio infinito en el valor de la resistividad superficial en una probeta de tamaño finito. Es decir, iguala el valor de la resistividad superficial medida en la probeta $\left(\rho_{s}\right)$ con la medida por el método volumétrico $\left(\rho_{\mathrm{v}}\right)$.

$$
F_{f}=\frac{\rho_{s}}{\rho_{\sin f}}=\frac{\rho_{v}}{\rho_{\sin f}}[9]
$$

donde $\rho_{\text {s,inf }}$ es la resistividad superficial-medio infinito. Los factores de forma para diversas geometrías se muestran en la Tabla 1.

Tabla 1. Factores de forma $\left(\mathrm{F}_{\mathrm{f}}\right)$ para diferentes tamaños de probeta y distancias entre electrodos

\begin{tabular}{|c|c|c|c|}
\hline $\begin{array}{c}\text { Separación electrodos } \\
\text { potencial }\end{array}$ & $\mathbf{a}=\mathbf{0 , 0 3 5 m}$ & $\mathbf{a = 0 . 0 3 7} \mathbf{~}$ & $\mathbf{a}=\mathbf{0 , 0 5 m}$ \\
\hline Cilíndrica $0,30 \times 0,15 \mathrm{~m}$ & 0,73 & 0,71 & 0,60 \\
\hline Cilíndrica $0,20 \times 0,10 \mathrm{~m}$ & 0,57 & & 0,38 \\
\hline Cilíndrica $0,15 \times 0,075 \mathrm{~m}$ & 0,38 & & \\
\hline Prismática $0,04 \times 0,04 \times 0,16 \mathrm{~m}$ & 0,17 & & 0,52 \\
\hline Cubos $0,15 \times 0,15 \times 0,15 \mathrm{~m}$ & & & \\
\hline
\end{tabular}

\section{DESCRIPCIÓN DEL MÉTODO OPERATORIO}

\subsection{Condiciones previas}

Cualquier resistivímetro comercial puede utilizarse para realizar las medidas, teniendo en cuenta los factores geométricos correspondientes. También es posible medir con un resistivímetro artesanal realizado a partir de una batería (corriente continua) y dos multímetros para medir potencial y corriente con una sonda artesanal para los cuatro electrodos (resistividad superficial) o con electrodos fabricados a partir de láminas metálicas (resistividad volumétrica).

El aparato seleccionado se calibra midiendo primero mediante un circuito preparado con resistencias eléctricas de valor conocido.

Si la medida se basa en el método volumétrico, no es necesaria ninguna calibración adicional. Si el método a usar es el superficial, primero se debe medir la resistividad volumétrica y luego la resistividad superficial. Esta calibración previa de la medida superficial permite obtener 
el factor de forma $\left(F_{f}\right)$ de la probeta concreta, según la ecuación [9].

El aparato debe ser capaz de aplicar una corriente estable (se recomienda que ésta sea alterna de hasta $40 \mathrm{~mA}$ ) entre los electrodos externos. También se podrá emplear una fuente de corriente alterna (con frecuencias entre 40 $\mathrm{Hz}$ y $2 \mathrm{KHz}$ ) o continua y dos multímetros para la determinación de la intensidad (I) y la tensión aplicada (V). El multímetro para medida del potencial debe ser de alta impedancia interna $\left(>10^{6} \Omega\right)$. Se podrá utilizar un registrador para la realización de varias medidas al mismo tiempo. Los valores mencionados en este apartado son solo orientativos.

Nota 1 - Puede utilizarse corriente continua o alterna indistintamente. Se recomienda que sea alterna para evitar una corrosión de los electrodos al aplicar la corriente siempre con la misma polaridad. La corriente alterna polariza lógicamente los electrodos igual que la continua, pero evita su corrosión. La corriente continua no se debe aplicar más allá de 5 segundos y la medida se toma a los 2-3 segundos.

Para la medida volumétrica se recomienda colocar una masa de alrededor de $2 \mathrm{Kg}$ encima de la probeta con el fin de presionar el conjunto y así se consiga buen contacto en todas las interfases. Entre los electrodos de corriente y el hormigón es necesario colocar esponjas húmedas que se ajusten al tamaño de los electrodos.

Los electrodos de potencial en el método volumétrico de 4 electrodos pueden ser abrazaderas metálicas de acero inoxidable o de cobre. Estas abrazaderas permiten tener la medida del potencial en todo el perímetro de la probeta a la vez.

Nota 2 Si la medida se hace con la probeta saturada de agua, no es necesario el interponer una esponja entre las abrazaderas y el hormigón, pero si la probeta no está saturada de agua, entonces es necesario colocar estas esponjas muy húmedas que faciliten el contacto electrolítico.

Si la medida se realiza sobre probetas saturadas de agua (curadas en balsa) se secará cuidadosamente su superficie lateral con un paño ligeramente húmedo para retirar el agua sobrante. No se recomienda la saturación a vacío pues cambia los valores de resistividad al sustituir la solución de los poros por el agua exterior, tanto si es agua potable, como si es hidróxido cálcico.

Nota $3 \mathrm{Si}$ el curado es en cámara húmeda, es necesario introducir la probeta totalmente sumergida en agua durante $24 \mathrm{~h}$ antes de realizar la medida.

La medida se realizará en una estancia con la temperatura adecuada de $20 \pm 2^{\circ} \mathrm{C}$. La probeta debe estar a esa temperatura en el momento de la medida.

Nota 4 Si la temperatura es diferente, se puede hacer la conversión a $20^{\circ} \mathrm{C}$ mediante la ley de Arrhenius siempre que la probeta esté saturada de agua o no varíe su humedad interior por la diferente temperatura.

$$
\rho(T)=\rho_{20} \cdot e^{\left\lfloor\frac{E_{a}}{R}\left(\frac{1}{T}-\frac{1}{T_{20}}\right)\right\rfloor}
$$

Donde $\rho(\mathrm{T})$ es la resistividad a la temperatura de la medida, $\rho_{20}$ es la temperatura a $20^{\circ} \mathrm{C}, \mathrm{R}$ es la constante de los gases 8,3143 (Julios $/ \mathrm{mol}^{\circ} \mathrm{K}$ ), $\quad \mathrm{T}_{20}$ es $293^{\circ} \mathrm{K}, \quad \mathrm{T}$ es la temperatura de la medida en ${ }^{\circ} \mathrm{K}$ y $\mathrm{E}_{\mathrm{a}}$ es la Energía de activación en Julios/mol. La Energía de Activación varía entre 15000 y 50000 Julios/mol. Se puede tomar como referencia un valor de 20000 Julios/mol.

Nota 4: Se indicará la temperatura de la sala de medición y de la balsa de curado en ${ }^{\circ} \mathrm{C}$.

\subsection{Realización de la medida}

\section{Método volumétrico}

La medida considera todo el volumen de la probeta (figura 1). Se colocan los electrodos frente-afrente con los que se aplica la corriente en dos caras paralelas de la probeta y mide el potencial en dos electrodos intermedios diferentes de los de corriente. 


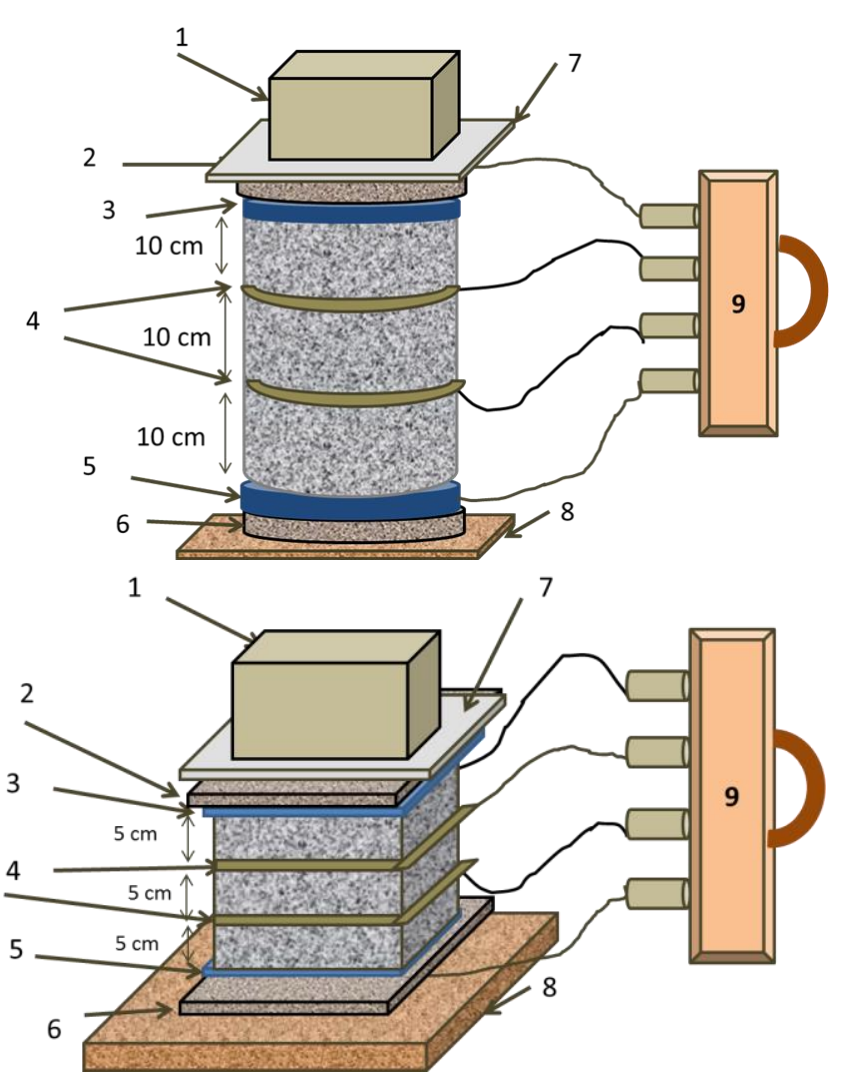

1. Masa para presionar y mejorar el contacto electrodo/esponja/hormigón

2. Electrodo superior para aplicar la corriente

3. Esponja

4. Abrazaderas metálicas (electrodos de potencial)

5. Esponja

6. Electrodo inferior de corriente

7. Lamina de plástico aislante

8. Material de soporte no conductor

9. Resistivímetro

Figura 1. Medida directa de la resistividad eléctrica.

Como indica la figura 1, se coloca un soporte aislante de la corriente. Sobre este soporte se coloca el electrodo inferior y sobre él, una esponja húmeda. Sobre este electrodo se coloca la probeta y sobre ella la otra esponja, el electrodo superior y la masa de unos $2 \mathrm{Kg}$ de peso para asegurar los contactos del conjunto.

A continuación, se efectúa la medida con el resistivímetro. Es necesario repetir la medida al menos una vez para confirmar que se repiten los resultados.

Nota 5 También se puede medir con solo los dos electrodos situados en las caras paralelas superior e inferior de la figura 1 conectando a ellos la medida de la corriente y el potencial. Este método con 2 electrodos es menos exacto porque existe una resistencia interfacial electrodo/esponja/hormigón que modifica el valor de la caída de potencial medida. Por ello se recomienda calibrar el método de 2 electrodos con el de 4 electrodos.
Nota 6 Se puede usar como resistivímetro el mismo que se usa para la medida del método Superficial. Para ello en la disposición de 4 electrodos se conectan las puntas exteriores que aplican la corriente a los dos electrodos superior e inferior del método volumétrico y las dos puntas interiores a los dos electrodos que miden el potencial como indica la figura 1. En la disposición de dos electrodos, es necesario cortocircuitar las puntas de corriente y voltaje de cada lado y conectarlos a los dos electrodos superior e inferior de la figura 1.

Nota 7 En el caso de usar un resistivímetro comercial para la medida de la resistividad volumétrica, es necesario tener en cuenta que hay que deducir de esa medida el valor de la resistencia $\left(\mathrm{R}_{\mathrm{e}}\right)$ dividiendo el valor de la resistividad por el factor $\mathrm{F}_{\mathrm{gs}}$ usado por el aparato (descrito en la ecuación [8]). A partir de la resistencia obtenida se recalcula la resistividad volumétrica utilizando el factor $\mathrm{F}_{\mathrm{gv}}$, según la ecuación [4].

\section{Método Superficial}

Es el método superficial se colocan los cuatro electrodos en la superficie de la probeta como indica la figura 2: los dos exteriores aplican la corriente y los dos interiores miden el cambio de potencial producido entre antes de aplicar la corriente y después. La distancia entre electrodos más idónea es la de $3,5 \mathrm{~cm}$ como máximo para probeta cilíndrica de $15 \times 30 \mathrm{~cm}$ o cúbica de $15 \times 15 \mathrm{~cm}$ con el fin de no acercarse al borde de la probeta. En la probeta cúbica se deben poner los electrodos en la diagonal como indica la figura 2.
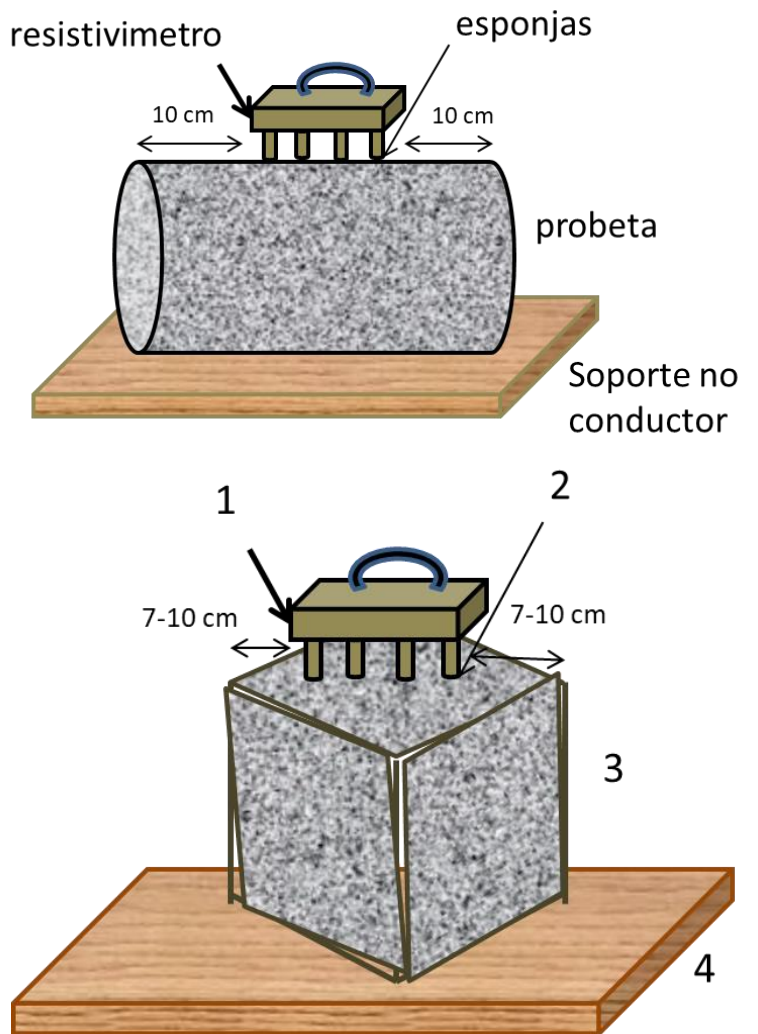

Figura 2. Medida Superficial de la resistividad. 
Si la probeta es cúbica se medirá en las 6 caras del cubo, o al menos en las 4 laterales y se hará la media, que será el resultado final.

Si la probeta es cilíndrica: se colocarán los 4 electrodos centrados respecto de las caras laterales en una generatriz de la probeta (figura 2). Debe haber una distancia al borde de la probeta mayor que la distancia entre sí de los electrodos.

Para medir a lo largo de todo el perímetro de forma homogéneamente espaciada, cuando se estabilice la corriente se realizarán seis medidas una sobre cada una de las seis generatrices separadas $60^{\circ}$ entre sí, alrededor de la muestra (figura 3). El valor de la resistividad eléctrica de cada probeta será la media de estas seis medidas.

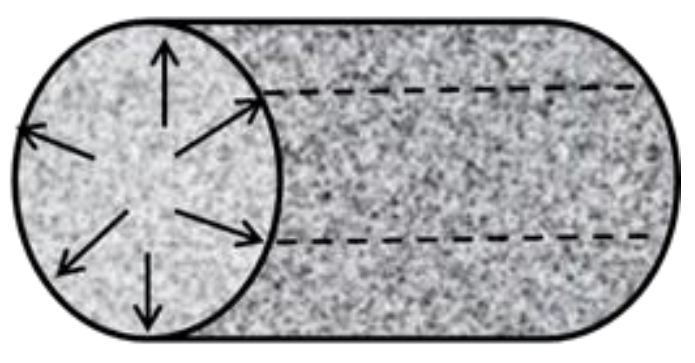

Figura 3. Las 6 generatrices en las que se debe tomar la medida de la resistividad superficial para hacer la media de la probeta

\section{CÁLCULOS}

\section{a. Método volumétrico}

En el caso de medir la resistividad volumétrica con una disposición de solo dos electrodos, (véase Nota 5 de la sección 4.2) la resistencia eléctrica medida debe corregirse restando la resistencia eléctrica de las esponjas húmedas, según la ecuación [11]. Una vez obtenido el valor de $R_{e}$ se aplicará la ecuación [4]

donde:

$$
\boldsymbol{R}_{e}=\boldsymbol{R}_{e+s p}-\boldsymbol{R}_{s p}
$$

- $\boldsymbol{R}_{e}$ es la resistencia eléctrica del hormigón (ecuación 1).

- $\boldsymbol{R}_{\boldsymbol{e}+s p}$ es la resistencia eléctrica del hormigón más la de las esponjas.

- $\boldsymbol{R}_{s p}$ es la resistencia eléctrica de las esponjas.

\section{b. Método Superficial}

Para el caculo de la resistividad se aplica la ecuación [6]

El Factor de forma $F_{f}$ para varias geometrías de probeta se muestra en la Tabla 1.

\section{PRECISION DEL ENSAYO}

En la Tabla 2 se muestran los Coeficientes de Variación de Repetibilidad y Reproducibilidad encontrados en dos ejercicios en 2016 y en 2019. 


\begin{tabular}{|c|c|c|}
\hline Ejercicio & $\begin{array}{c}\text { Repetibilidad } \\
\text { Coeficiente de variación } \\
\text { (C.V-\%) }\end{array}$ & $\begin{array}{c}\text { Reproducibilidad } \\
\text { Coeficiente de variación } \\
\text { (C.V-\%) }\end{array}$ \\
\hline $\begin{array}{c}\text { Instituto de Ciencias de la } \\
\text { Construcción "Eduardo } \\
\text { Torroja"-2016 } \\
\text { (4 técnicos diferentes) }\end{array}$ & 2,46 & 5,15 \\
\hline $\begin{array}{c}\text { Ensayo Interlaboratorios } \\
\text { "CALIDUR"-2019 } \\
(22 \text { laboratorios) }\end{array}$ & 3,37 & 13,56 \\
\hline
\end{tabular}

\section{INFORME}

El informe de la determinación de la resistividad eléctrica en probetas deberá contener la siguiente información:

- Nombre y dirección del responsable de las medidas.

- Fecha de la realización de las medidas.

- Fecha de realización del informe.

- Nombre y dirección del cliente.

- Descripción y foto de las probetas.

- Temperatura y humedad relativa del laboratorio.

- Método de ensayo utilizado e identificación del resistivímetro.

- En el caso de probetas fabricadas en laboratorio indicar el método de curado o método de acondicionamiento de la humedad de las probetas.

- En el caso de testigos de hormigón extraídos de obra construida indicar el método de acondicionamiento de la humedad de los testigos

- Resultados de resistividad

- Firma y titulación, u otra identificación de la/s persona/s responsable/s del contenido técnico del informe.

\section{BIBLIOGRAFÍA}

1. Wenner, F.: "A Method for Measuring Earth Resistivity", Bureau of Standards J., 12 (1915) $469-478$.

2. ASTM G-57-84: "Standard Method for Field Measurement of Soil Resistivity using the Wenner four-electrode Method", American Society for Testing and Materials, West Conshohocken, PA, USA (2001).

3. NBR 9204. Concreto Endurecido. "Determinaçâo da resistividade elétrica volumétrica". Asociación Brasileira de Normas Técnicas (ABNT). Río de Janeiro-Brasil, 1985. 
4. Millard, S. G.; Harrison, J.A. and Edwards, A.J.: "Measurements of Electrical Resistivity of Reinforced Concrete Structures for the Assessment of Corrosion Risk". British Journal of NTD, 31 (1989) 617 - 621.

5. Polder, R.; Andrade, C.; Elsener, B.; Vennesland, O.; Gulikers, J.; Weidert, R. and Raupach, M.: " Test methods for on-site measurement of resistivity of concrete", RILEM TC 154-EMC: Electrochemical techniques for measuring metallic corrosion", Materials and Structures, 33 (2000) 603-611.

6. Morris, W.; Moreno, E.I. and Sagüés, A.A.: "Practical evaluation of resistivity of concrete in test cylinders using a Wenner array probe”, Cement and Concrete Research, 26 (1996) 17791787.

7. Andrade C.; Fullea J. and Alonso C.: "The use of the graph corrosion rate-resistivity in the measurement of the corrosion current". Proceedings of the International Workshop on "Measurement and interpretation of the on-site corrosion rate"-. MESINA- RILEM Proc. No. 18. (Eds.: C. Andrade, C. Alonso, J. Fullea, J. Polimon and J. Rodriguez). RILEM PUBLICATIONS S.A.R.L. (2000) 157-166.

8. Andrade, C.; Castellote, M. and D'Andrea, R.: "Measurement of ageing effect of chloride diffusion coefficients in cementitious matrices·, Journal of Nuclear Materials, 412 (2011) 209216. 